\title{
NBD-Based Environment-Sensitive Fluorescent Probes for the Human Ether-a-Go-Go-Related Gene Potassium Channel
}

\author{
Qi $\mathrm{Li}^{1 \dagger}$, Lijuan Chai ${ }^{2 \dagger}$, Gaopan Dong ${ }^{1}$, Xiaomeng Zhang ${ }^{1}$ and Lupei $\mathrm{Du}^{1 *}$ \\ ${ }^{1}$ Department of Medicinal Chemistry, Key Laboratory of Chemical Biology (MOE), School of Pharmacy, Shandong University, \\ Jinan, China, ${ }^{2}$ Department of Internal Medicine, Hospital of Shandong University, Jinan, China
}

Three environment-sensitive probes were developed for the hERG channel based on the nitrobenzoxadiazole fluorophore herein. After careful evaluation, probes M1 and M3 were found to have a high affinity for imaging the hERG channel in the cell-based experiment. Compared with other fluorescent labeling technologies (such as fluorescent proteins), these probes afford a convenient and economical method to determine hERG channel in vitro and in cellulo. Therefore, these probes are expected to be applicable for usage in physiological and pathological studies of hERG channels and have the potential to establish a screening system for hERG channels.

Keywords: hERG potassium channel, fluorescent labeling technology, small-molecule fluorescent probe, fluorescent imaging, cell imaging

\section{INTRODUCTION}

The human ether-a-go-go-related gene ( $\mathrm{hERG}$ ) codes to form a rapidly delayed rectifying potassium ion channel. The channel consists of four identical alpha subunits forming a tetrameric structure and a pore-forming region in the middle. Each subunit consists of six helical transmembrane domains (denoted as S1-S6) (Doyle et al., 1998). In cardiomyocytes, loss of hERG function is one of the causes of acquired long QT interval syndrome, and acquired functional mutation may also cause short QT interval syndrome (Moss et al., 2002). At present, many drugs that are on the market or in the clinical stage have prolonged the QT interval due to their inhibitory effect on the hERG channel and are withdrawn from the market. Prolonged QT interval displays delayed ventricular repolarization, which can cause torsades de pointes (TdP), thus leading to cardiotoxicity. Therefore, the hERG potassium channel is currently the main anti-target. At the FDA's request, all drugs need to be tested for their affinity for hERG channels to assess their cardiotoxicity (Raschi et al., 2008; Liu et al., 2016).

The hERG potassium channel distributes in various cancer cells, neurons, smooth muscle cells of organs, and chromaffin cells, performing different roles in different tissues (Raschi et al., 2008). Over the past 2 decades, there is increasing evidence that the hERG potassium channel abnormally expresses in tumor cell lines and primary human cancers, such as gliomas, neural crest-derived tumors. Cell and molecular studies indicate that the hERG channel regulates different aspects of tumor progression, such as cell proliferation and survival, secretion, invasion, and metastasis of proangiogenic factors (Jehle et al., 2011). Therefore, the hERG potassium channel may be the target for particular cancer treatment. To better analyze and determine the hERG potassium channel at the molecular and cellular level, developing a simple, cost-effective, and safe fluorescent method for labeling the hERG potassium channel is urgently required (Luo et al., 2020). Effective development of 


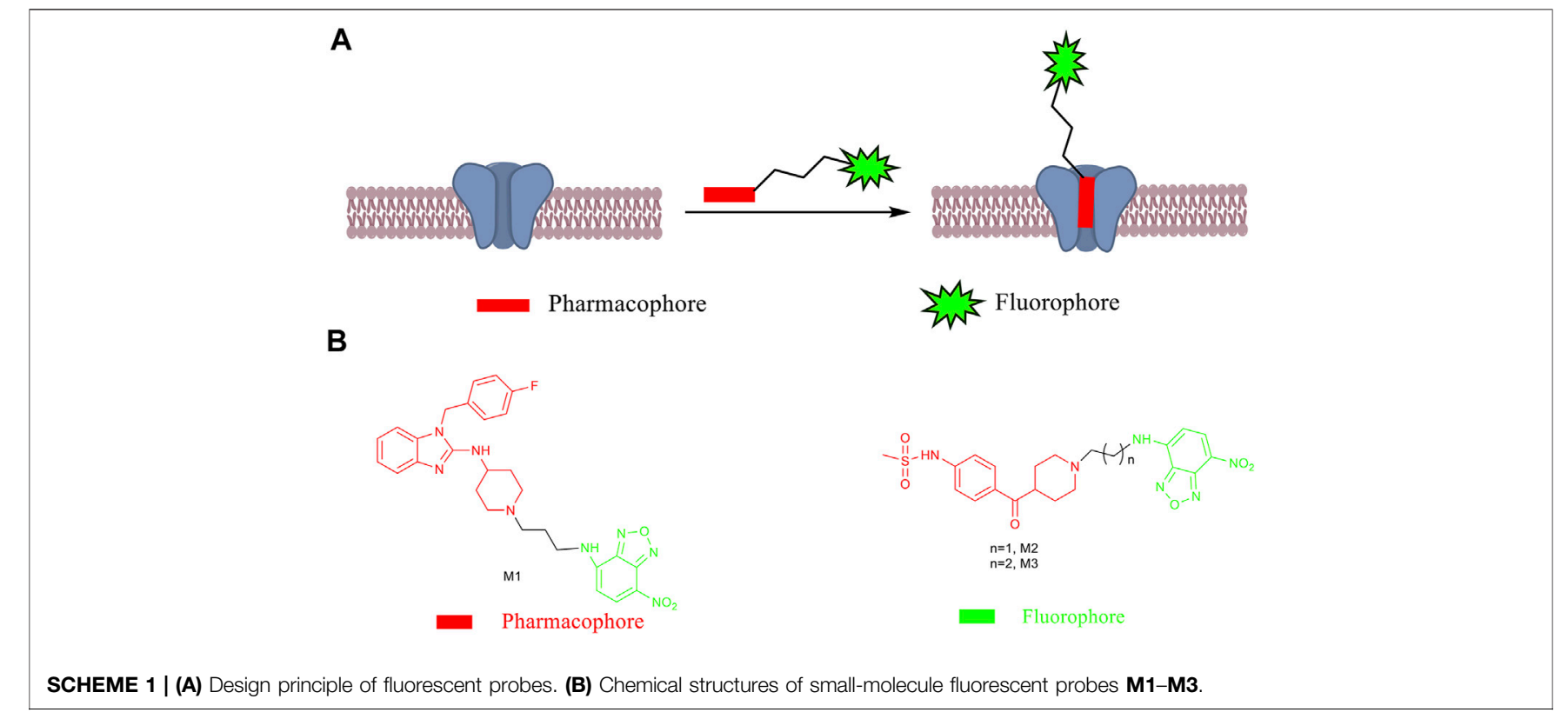

SCHEME 1 | (A) Design principle of fluorescent probes. (B) Chemical structures of small-molecule fluorescent probes $\mathbf{M 1 - M 3}$
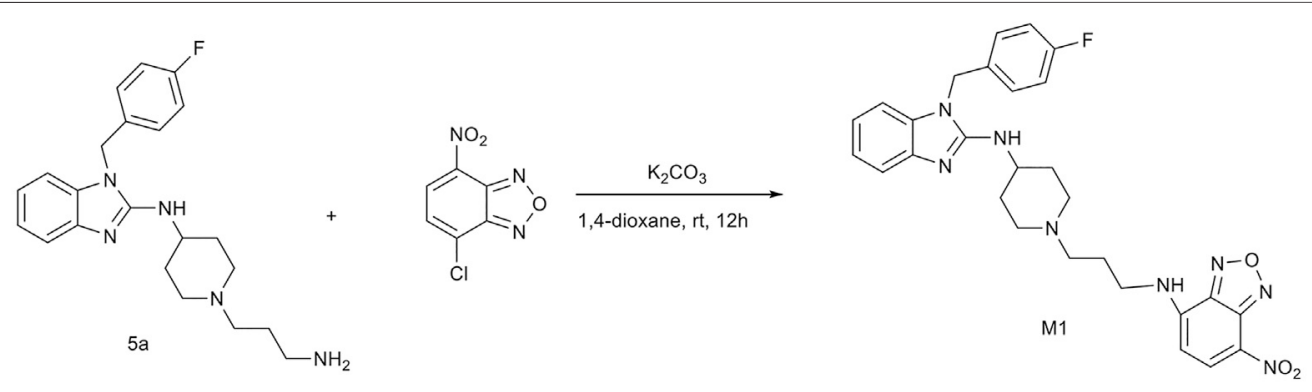

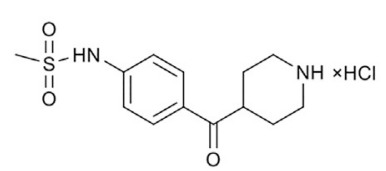

$2 b$

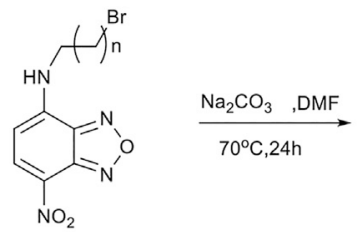

$\mathrm{n}=1,1 \mathrm{~d}$ $n=2,2 d$

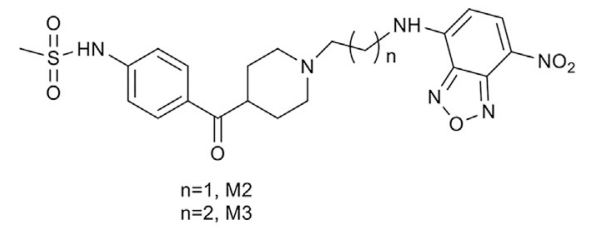

FIGURE 1 | Synthetic routes of fluorescent probes M1, M2, and M3.

such fluorescent labeling may explain the hERG channel's role in those cancers and anatomical locations, thus establishing the hERG channel as a brand new target and/or biomarker for antitumor agents.

The fluorescent labeling technologies for the hERG potassium channel mainly rely on small-molecule fluorescent probes and fluorescent protein-based labeling technologies (Claassen et al., 2008; Huang et al., 2011; Mizukami and Kikuchi, 2016; Huang et al., 2020). With the unique characteristics such as high sensitivity, low detection limit, simple preparation, and low cost, the application of small-molecule fluorescent probe is available for detecting and imaging enzymes, receptors, ion channels, DNA, RNA, and biologically active small molecules $\left(\mathrm{H}_{2} \mathrm{~S}, \mathrm{H}_{2} \mathrm{O}_{2}\right.$, etc.) directly, or
TABLE 1 | Optical properties of probes M1, M2, and M3

\begin{tabular}{lccccc} 
Probes & $\lambda_{\text {max }}$ (nm) & $\boldsymbol{\varepsilon}$ (L/mol.cm) & $\lambda_{\text {ex }}(\mathbf{n m})$ & $\lambda_{\text {em }}$ (nm) & $\Phi(\%)$ \\
\hline M1 & 475 & $6.06 \times 10^{4}$ & 475 & 545 & 9.40 \\
M2 & 465 & $3.07 \times 10^{4}$ & 470 & 530 & 7.31 \\
M3 & 470 & $5.10 \times 10^{4}$ & 475 & 545 & 10.1
\end{tabular}

for even tracking dynamic processes in a real-time manner at the animal level (Chan et al., 2012; Mizukami et al., 2013).

After the fluorescent probe binds to the target, the fluorophore converts the interaction between the recognition and target 

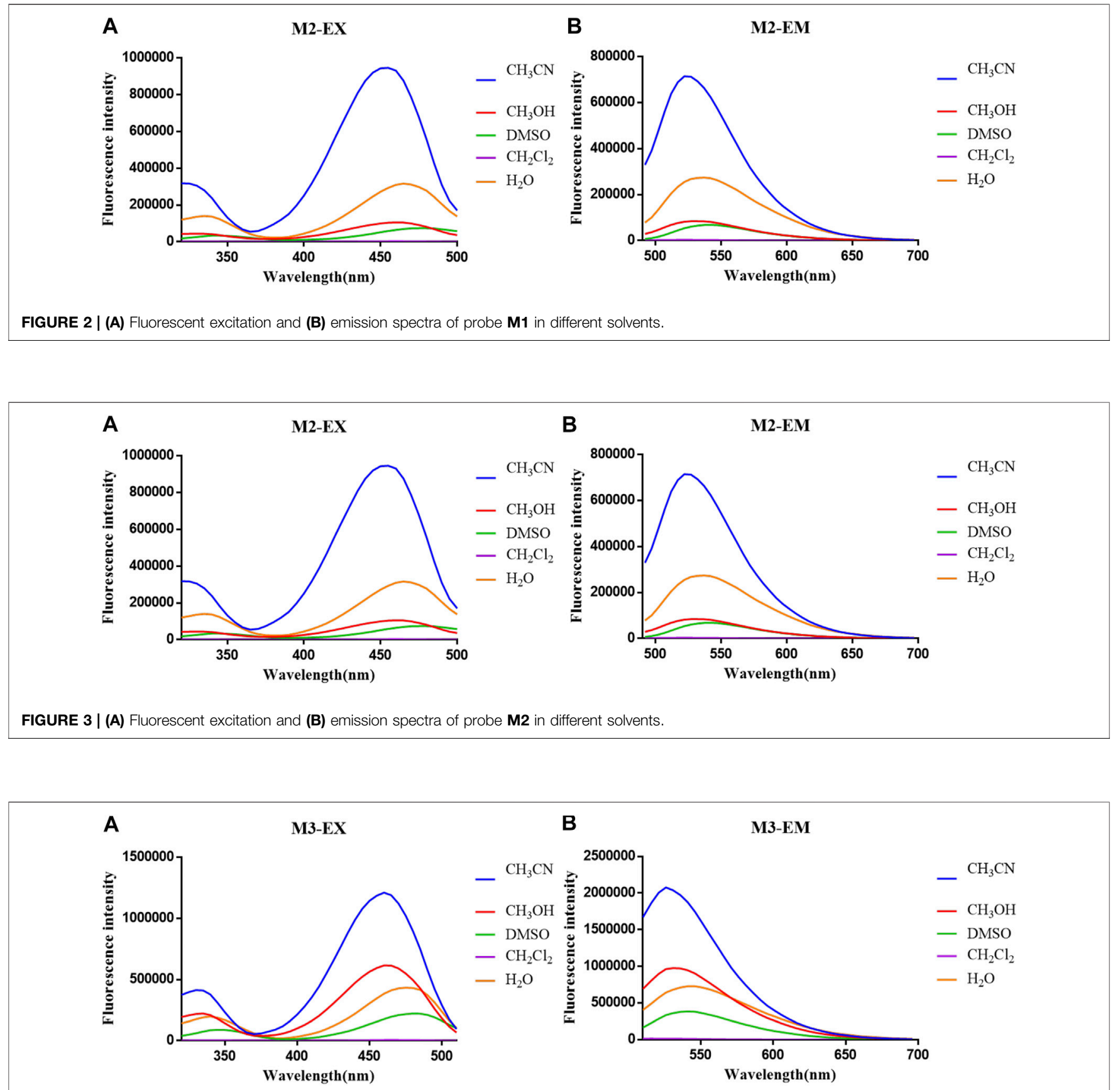

FIGURE 4 | (A) Fluorescent excitation and (B) emission spectra of probe M3 in different solvents.

groups into a fluorescent signal. In this process, the excess unbound fluorescent probe has a specific effect on target binding and fluorescence detection (Mizukami et al., 2013). An inevitable turn-on switch is usually incorporated into the design of the probe. It is known that the principal mode of action between ligands and the hERG potassium channel is through hydrophobic interaction with Tyr 652 and/or Phe 656 residues. Therefore, we suspect that an environment-sensitive fluorophore, such as 6-dimethylaminonaphthalene (DAN), 1,8- anilinonaphthalenesulfonic acid (ANS), nitrobenzoxadiazole (NBD), and sulfonyl benzoxadiazole (SBD), can be integrated into the probe design (Environment-Sensitive Fluorescent Probe for the Human Ether-a-go-go-Related Gene Potassium Channel, Environment-Sensitive Fluorescent Turn-On Probes Targeting Hydrophobic Ligand-Binding Domains for Selective Protein Detection, Kilpin et al., 2012; Zhuang et al., 2013). Considering that the probe's bulky size may affect the affinity with the target, we chose a relatively small NBD fluorophore to participate in the 


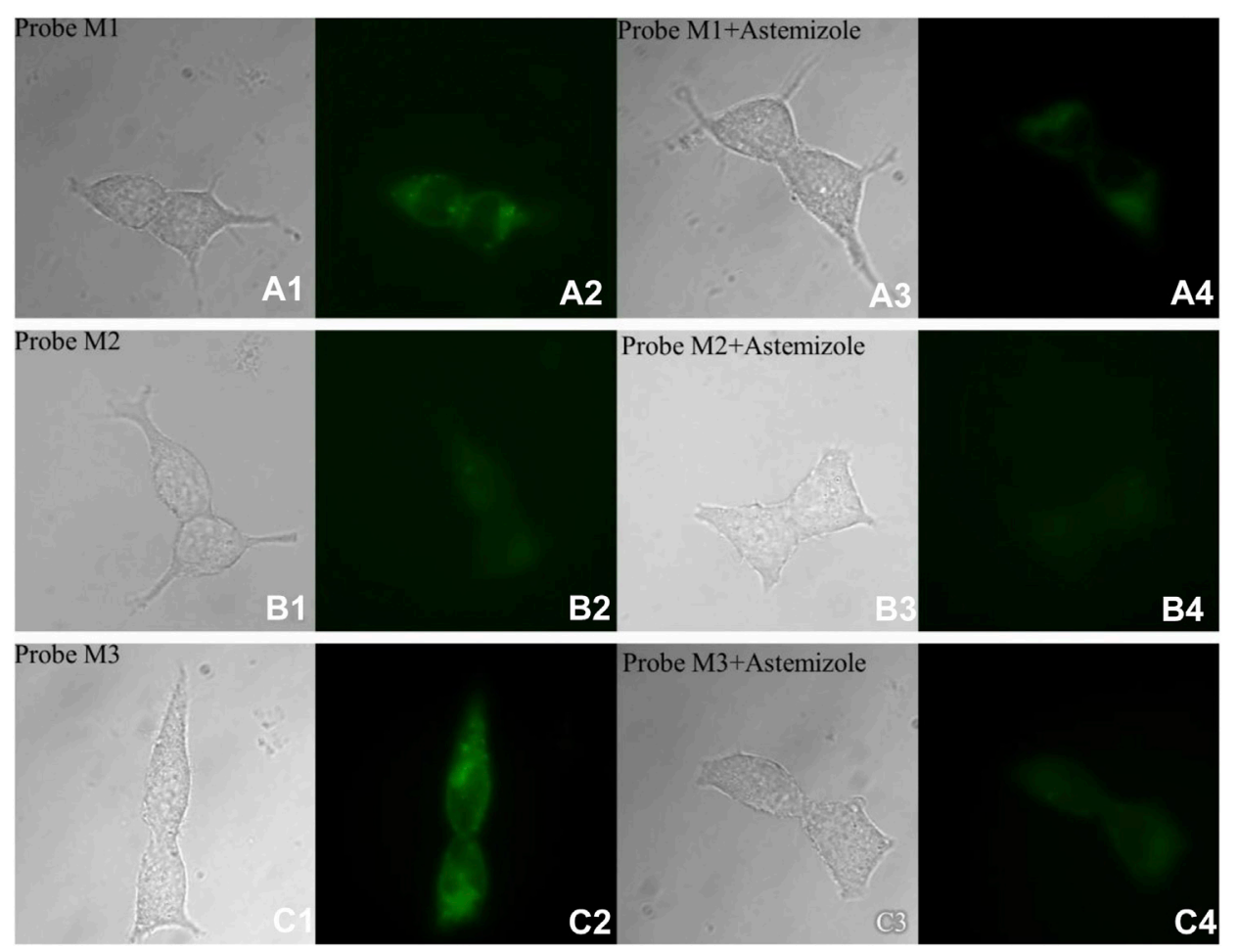

FIGURE 5 | Fluorescence microscopy imaging of hERG-transfected HEK293 cells incubated with 1.0 $\mu$ M probe M1 (A1: bright field; A2: GFP channel), $5.0 \mu$ M probe M2 (B1: bright field; B2: GFP channel), and $5.0 \mu \mathrm{M}$ probe $\mathbf{M 3}$ (C1: bright field; C2: GFP channel). The imaging of inhibition of the hERG channels was

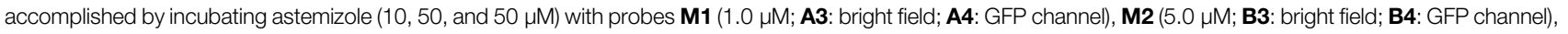
and $\mathbf{M 3}$ (5.0 $\mu \mathrm{M}$; C3: bright field; C4: GFP channel). All cells were incubated with each probe at $37^{\circ} \mathrm{C}$ for 10 min and washed immediately. The background was adjusted by ImageJ software. Imaging was performed using a Zeiss Axio Observer A1 microscope with a ×63 objective lens. Scale bar = $20 \mu \mathrm{m}$.

hydrophobic interaction with the hERG channel as a part of the recognition group (Ma et al., 2016; Perez et al., 2019).

\section{RESULTS AND DISCUSSION}

\section{Design and Synthesis of Fluorescent Probes}

Astemizole and E-4031 are both very effective drugs with inhibitory effects on the hERG channel (Zhou et al., 1999; Tian et al., 2017; Dickson et al., 2020). Studies on the mechanism of action between the hERG potassium channel and inhibitors have revealed that the high-affinity binding of hERG channels requires the inhibitor's aromatic group and basic nitrogen. Based on the above, the major interacting parts of astemizole and E-4031 were selected as the recognition group.

As depicted in Scheme 1 and Figure 1, probes M1, M2, and M3 were well designed and synthesized through several convenient steps. In brief, the fluorophore $\mathrm{NBD}-\mathrm{Cl}$ and astemizole-based intermediate 5 a produced probe $\mathbf{M 1}$ in the presence of $\mathrm{K}_{2} \mathrm{CO}_{3}$ in 1,4-dioxane. NBD-Cl derivatives $\mathbf{1 d}$ and $\mathbf{2 d}$ reacted with E-4031-based intermediate $\mathbf{2 b}$ to provide probes M2 and $\mathbf{M 3}$ in DMF in the presence of $\mathrm{NaCO}_{3}$. Detailed information on synthetic intermediates can be found in the supporting information.
TABLE 2 | Cytotoxicity of fluorescent probes for hERG-transfected HEK293.

Probes

$\mathrm{IC}_{50}(\mu \mathrm{M})$

hERG-HEK 293

\begin{tabular}{lc}
\hline Astemizole & $17.4 \pm 1.06$ \\
M1 & $10.6 \pm 2.74$ \\
M2 & $>100$ \\
M3 & $>100$
\end{tabular}

\section{Spectroscopic Properties of Fluorescent Probes}

The optical properties of probes M1, M2, and M3 were measured in a $10 \mu \mathrm{M}$ PBS solution $(\mathrm{pH}=7.4)$. The experimental results display that these probes have rational optical properties (Table 1). Moreover, the varied fluorescence intensity in different solvents indicates the environment-sensitive effect caused by the NBD-Cl fluorophore (Figures 2-4).

\section{Cytotoxicity Assay of Fluorescent Probes}

The cytotoxicity of probes M1-M3 for hERG-transfected HEK293 cells was evaluated by an SRB assay. The experimental results showed that these probes have low cytotoxicity, have little effect on the viability of living cells, and can be used to detect and image hERG potassium channels in living cells (Table 2). 
TABLE 3 | Affinity of probes for hERG-transfected HEK293.

\begin{tabular}{lcc}
\hline Molecule & $\mathbf{I C}_{\mathbf{5 0}}{ }^{\mathbf{a}} \mathbf{( n M )}$ & $\mathbf{K}_{\mathbf{i}}^{\mathbf{b}}(\mathbf{n M})$ \\
\hline Astemizole & 4.76 & 2.67 \\
M1 & 6.75 & 3.79 \\
M2 & 67.4 & 37.8 \\
M3 & 10.8 & 6.08
\end{tabular}

${ }^{a}$ See the Supporting Information.

${ }^{b}$ The inhibition constant (Ki) was calculated from each $I C_{50}$ value using the Cheng-Prusoff equation.

TABLE 4 | Fluorescence intensity of cell imaging.

\begin{tabular}{lcc} 
Molecule & Mean $^{\mathbf{a}}$ (probe) & Mean $^{\mathbf{a}}$ (probe + astemizole) \\
\hline M1 & 51.076 & 34.666 \\
M2 & 34.988 & 28.353 \\
M3 & 67.015 & 26.213
\end{tabular}

${ }^{a}$ Mean: Mean gray value; Mean $=$ IntDen/Area

\section{Binding Affinity of Fluorescent Probes}

Cell membranes extracted from hERG-transfected HEK293 cells were evaluated for probes' affinity to the hERG channel by a radioligand binding assay. The binding results demonstrated that probe M1 exhibited a high affinity for $h E R G$ potassium channels. The calculated $\mathrm{IC}_{50}$ and $\mathrm{K}_{\mathrm{i}}$ values were 6.75 and $3.79 \mathrm{nM}$, respectively, slightly lower than those for astemizole $(4.76$ and $2.67 \mathrm{nM}$, respectively). The affinity of probe M3 for the hERG channel is similar to that of M1, with $\mathrm{IC}_{50}$ of $10.8 \mathrm{nM}$ and $\mathrm{K}_{\mathrm{i}}$ of $6.08 \mathrm{nM}$. Although having lower activity than probe $\mathbf{M} 1$ and astemizole, probe M2 still displays a potent affinity for the hERG channel (Table 3).

\section{Fluorescent Imaging Studies}

Considering their sufficient affinity for $\mathrm{hERG}$ potassium channel, good fluorescent properties, and suitable cytotoxicity, probes M1-M3 were applied for labeling the hERG channel in living cells. Initially, the autofluorescence of hERG-HEK293 cells was well evaluated. The experimental results indicated that the cell autofluorescence is too weak to be measured with or without astemizole (Supplementary Figure S2). Therefore, this weak cell autofluorescence would not interfere with the labeling of the hERG channel by probes M1-M3.

In cell imaging, probe M1 can achieve high brightness at low concentrations $(0.1 \mu \mathrm{M})$, consistent with its affinity from the radioligand binding experiment. The reason why probes M2 and M3 require high concentrations $(5 \mu \mathrm{M})$ is that the pharmacophore of probes $\mathbf{M} 2$ and $\mathbf{M} 3$ is E-4031, which has a slightly lower affinity for hERG channels than astemizole. Moreover, a potent hERG channel inhibitor (astemizole) was selected to incubate cells with each probe. After incubation with astemizole, the fluorescence intensity of all probes decreased significantly (Figure 5, Table 4).

\section{CONCLUSION}

In conclusion, we designed, synthesized, and evaluated three small-molecule fluorescent probes herein. Introduction of the environment-sensitive fluorophore NBD revealed that these probes do not require a time-consuming washing procedure during microscopic imaging. Compared with other reported fluorescent labeling technologies, such as fluorescent proteins, these probes provide a convenient and affordable method for detecting the hERG channel. It should be noted that among all probes, molecules $\mathbf{M 1}$ and $\mathbf{M} 3$ have higher affinity and more vigorous fluorescence intensity than our previously published environment-sensitive probes (Environment-Sensitive Fluorescent Probe for the Human Ether-a-go-go-Related Gene Potassium Channel, Wang et al., 2016). However, more challenge remains to be undertaken, especially in developing near-infrared probes for hERG channel for in vivo research.

\section{DATA AVAILABILITY STATEMENT}

The datasets presented in this study can be found in online repositories. The names of the repository/repositories and accession number(s) can be found in the article/ Supplementary Material.

\section{AUTHOR CONTRIBUTIONS}

QL, LC, and LD conceived and designed the experiments. QL, LC, and GD performed the experiments. QL, LC, GD, and XZ contributed to writing the manuscript. All authors read and approved the manuscript.

\section{FUNDING}

The present project was supported by grants from the Shandong Natural Science Foundation (No. ZR2017MH101) and the Key Research and Development Project of Shandong Province (No. 2017CXGC1401).

\section{ACKNOWLEDGMENTS}

The manuscript was written through contributions of all authors.

\section{SUPPLEMENTARY MATERIAL}

The Supplementary Material for this article can be found online at: https://www.frontiersin.org/articles/10.3389/fmolb.2021.666605/ full\#supplementary-material 


\section{REFERENCES}

Chan, J., Dodani, S. C., and Chang, C. J. (2012). Reaction-based Small-Molecule Fluorescent Probes for Chemoselective Bioimaging. Nat. Chem. 4, 973-984. doi:10.1038/nchem.1500

Claassen, S., Schwarzer, S., Ludwig, J., and Zünkler, B. J. (2008). Electrophysiological and Fluorescence Microscopy Studies with HERG Channel/EGFP Fusion Proteins. J. Membr. Biol 222, 31-41. doi:10.1007/s00232-008-9101-0

Dickson, C. J., Velez-Vega, C., and Duca, J. S. (2020). Revealing Molecular Determinants of hERG Blocker and Activator Binding. J. Chem. Inf. Model. 60, 192-203. doi:10.1021/acs.jcim.9b00773

Doyle, D. A., Cabral, J. M., Pfuetzner, R. A., Kuo, A., Gulbis, J. M., Cohen, S. L., et al. (1998). The Structure of the Potassium Channel: Molecular Basis of K+ Conduction and Selectivity. Science 280, 69-77. doi:10.1126/science.280.5360.69

Huang, N., Lian, J. F., Huo, J. H., Liu, L. Y., Ni, L., Yang, X., et al. (2011). The EGFP/ hERG Fusion Protein Alter the Electrophysiological Properties of hERG Channels in HEK293 Cells. Cell. Biol. Int. 35, 193-199. doi:10.1042/ cbi20100022

Huang, Y., Lv, T., Qin, T., Xu, Z., Wang, L., and Liu, B. (2020). A DS2-specific Flavonoid-Based Probe with a Unique Dual-Emissive Response to Human Serum Albumin. Chem. Commun. 56, 11094-11097. doi:10.1039/d0cc04359a

Jehle, J., Schweizer, P. A., Katus, H. A., and Thomas, D. (2011). Novel Roles for hERG K+ Channels in Cell Proliferation and Apoptosis. Cell Death Dis 2, e193. doi:10.1038/cddis.2011.77

Kilpin, K. J., Clavel, C. M., Edafe, F., and Dyson, P. J. (2012). NaphthalimideTagged Ruthenium-Arene Anticancer Complexes: Combining Coordination with Intercalation. Organometallics 31, 7031-7039. doi:10.1021/om3007079

Liu, Z., Jiang, T., Wang, B., Ke, B., Zhou, Y., Du, L., et al. (2016). Environment-Sensitive Fluorescent Probe for the Human Ether-A-Go-Go-Related Gene Potassium Channel. Anal. Chem. 88, 1511-1515. doi:10.1021/acs.analchem.5b04220

Luo, Z., Lv, T., Zhu, K., Li, Y., Wang, L., Gooding, J. J., et al. (2020). Paper-Based Ratiometric Fluorescence Analytical Devices towards Point-of-Care Testing of Human Serum Albumin. Angew. Chem. 132, 3155-3160. doi:10.1002/ange. 201915046

Ma, Z., Lin, Y., Chen, H., Du, L., and Li, M. (2016). A Novel NBD-Based pH "On-Off" Fluorescent Probe Equipped with the N-Phenylpiperazine Group for Lysosome Imaging. RSC Adv. 6, 102773-102777. doi:10.1039/c6ra16996a

Mizukami, S., Hori, Y., and Kikuchi, K. (2013). Small-Molecule-Based ProteinLabeling Technology in Live Cell Studies: Probe-Design Concepts and Applications. Acc. Chem. Res. 47, 247-256. doi:10.1021/ar400135f
Mizukami, S., and Kikuchi, K. (2016). Visualization and Functional Regulation of Live Cell Proteins Based on Labeling Probe Design. Yakugaku Zasshi 136, 21-27. doi:10.1248/yakushi.15-00225-4

Moss, A. J., Zareba, W., Kaufman, E. S., Gartman, E., Peterson, D. R., Benhorin, J., et al. (2002). Increased Risk of Arrhythmic Events in Long-QT Syndrome with Mutations in the Pore Region of the Human Ether-A-Go-Go-Related Gene Potassium Channel. Circulation 105, 794-799. doi:10.1161/hc0702. 105124

Pérez, A. L., Tibaldo, G., Sánchez, G. H., Siano, G. G., Marsili, N. R., and Schenone, A. V. (2019). A Novel Fluorimetric Method for Glyphosate and AMPA Determination with NBD-Cl and MCR-ALS. Spectrochimica Acta A: Mol. Biomol. Spectrosc. 214, 119-128. doi:10.1016/j.saa.2019.01.078

Raschi, E., Vasina, V., Poluzzi, E., and De Ponti, F. (2008). The hERG K+ Channel: Target and Antitarget Strategies in Drug Development. Pharmacol. Res. 57, 181-195. doi:10.1016/j.phrs.2008.01.009

Tian, J., Vandermosten, L., Peigneur, S., Moreels, L., Rozenski, J., Tytgat, J., et al. (2017). Astemizole Analogues with Reduced hERG Inhibition as Potent Antimalarial Compounds. Bioorg. Med. Chem. 25, 6332-6344. doi:10.1016/j. bmc.2017.10.004

Wang, B., Liu, Z., Ma, Z., Li, M., and Du, L. (2016). Astemizole Derivatives as Fluorescent Probes for hERG Potassium Channel Imaging. ACS Med. Chem. Lett. 7, 245-249. doi:10.1021/acsmedchemlett.5b00360

Zhou, Z., Gong, Q., and January, C. T. (1999). Correction of Defective Protein Trafficking of a Mutant HERG Potassium Channel in Human Long QT Syndrome. J. Biol. Chem. 274, 31123-31126. doi:10.1074/jbc.274.44.31123

Zhuang, Y.-D., Chiang, P.-Y., Wang, C.-W., and Tan, K.-T. (2013). EnvironmentSensitive Fluorescent Turn-On Probes Targeting Hydrophobic Ligand-Binding Domains for Selective Protein Detection. Angew. Chem. 125, 8282-8286. doi:10. 1002/ange.201302884

Conflict of Interest: The authors declare that the research was conducted in the absence of any commercial or financial relationships that could be construed as a potential conflict of interest.

Copyright (c) $2021 \mathrm{Li}$, Chai, Dong, Zhang and Du. This is an open-access article distributed under the terms of the Creative Commons Attribution License (CC BY). The use, distribution or reproduction in other forums is permitted, provided the original author(s) and the copyright owner(s) are credited and that the original publication in this journal is cited, in accordance with accepted academic practice. No use, distribution or reproduction is permitted which does not comply with these terms. 